\title{
Physicochemical properties and sensory attributes of medium- and long-chain triacylglycerols (MLCT)-enriched bakery shortening.
}

\begin{abstract}
Six binary formulations of medium- and longchain triacylglycerols (MLCT) fat and palm stearin and four ternary formulations of MLCT fat, palm stearin, and palm olein were produced. MLCT fat and palm stearin were mixed in ranges of $40-90 \%$ with $10 \%$ increments (w/w), while for the ternary formulations, $10 \%$ and $20 \%$ palm olein were substituted to palm stearin in MS 46 and MS 55 formulations. The solid fat content (SFC) by pulsed nuclear magnetic resonance and heating profiles using differential scanning calorimeter of these formulations were determined. Results obtained from SFC and heating profiles found that all the formulations melted completely at $55{ }^{\circ} \mathrm{C}$. The high complete melting temperature is due to the stearic acid content in MLCT fat. Generally, increasing \% MLCT fat (40-90\%) in the formulations lowers the SFC curves at the measured temperatures $\left(0-60{ }^{\circ} \mathrm{C}\right)$. The binary samples of MS 73, MS 82, and MS 91 showed SFC between $15 \%$ and $25 \%$ at room temperature $\left(25{ }^{\circ} \mathrm{C}\right)$, which indicated that these formulations were suitable for shortening production. As the production cost of MLCT fat is high (approximately USD 3/kg), an attempt to reduce the proportion of MLCT fat in the shortening formulations was done by developing the ternary formulations. Shortenings formulated with 40:40:20 (MSO 442), 50:40:10 (MSO 541), and 50:30:20 (MSO 532) of MLCT fat/palm stearin/palm olein formulations had similar SFC\% at $25{ }^{\circ} \mathrm{C}$, and they were subsequently chosen to produce shortening. Using multivariate analysis, taste attribute showed positively and highly correlated to the melting temperature and SFC at $25{ }^{\circ} \mathrm{C}$ of the LCT-enriched shortenings. In acceptance test, high correlation $(\mathrm{R} 2=0.98)$ was only found on cakes made from MSO 442 and MSO 541 shortenings. Both untrained and trained panelists rated the Madeira cakes made from MSO 532 shortening the highest for overall acceptability.
\end{abstract}

Keyword: Shortening; Medium-and long-chain triacylglycerol; Solid fat content; Heating profiles. 